\title{
X-type interactions of the loops in the flare of 25 September 1997 - Part II
}

\author{
P. Rudawy ${ }^{1}$, R. Falewicz ${ }^{1}$, C. H. Mandrini ${ }^{2}$, and M. Siarkowski ${ }^{3}$ \\ 1 Astronomical Institute of the Wrocław University, ul. Kopernika 11, 51-622 Wrocław, Poland \\ 2 Instituto de Astronomía y Física del Espacio, CC. 67 Suc. 28, 1428 Buenos Aires, Argentina \\ 3 Solar Physics Division, Space Research Centre, ul. Kopernika 11, 51-622 Wrocław, Poland
}

Received 6 February 2001 / Accepted 29 March 2001

\begin{abstract}
The interaction of magnetic structures and the conversion of magnetic free energy into heating of the plasma and acceleration of particles are commonly accepted as the origin of solar flares. The 3-dimensional (3D) structure and evolution of the coronal magnetic field can be investigated indirectly by the analysis of the images of various loops filled with emitting plasma or/and through a numerical modelling based on photospheric field measurements. Exploring multi-wavelength observations of the C7.2 class solar flare that occurred in the NOAA 8088 active region (AR) on 25 September 1997, we have previously built (Falewicz \& Rudawy 1999, hereafter Paper I) a phenomenological 3D model of the magnetic configuration of the flare area, and a scenario of its evolution. In this paper, we present the results of a numerical modelling of the magnetic field of the NOAA 8088 active region. Taking into account all the limitations of this approach, we describe probable scenarios of the magnetic field evolution before and during the flare. We found that construction of the realistic model of the flare and its evolution requires the comparison of the numerical model with the results of the analysis of various observational data. After doing this, we still find that the correct interpretation of the numerical results is difficult and can be considered as a typical problem. Using X-ray data taken with the RF15-I instrument on board the INTERBALL-TAIL satellite, we extended our previous analysis of the relation between the hard X-ray and microwave emissions during the flash phase of the flare. We found that all the recorded pulses of the hard $\mathrm{X}$-ray and $3 \mathrm{GHz}$ microwave fluxes showed a tendency to quasi-periodical repetition and very similar temporal changes of the signals. The calculated temperatures of the flare plasma indicate that the pre-maximum peaks of the hard X-rays and microwaves were generated during a non-thermal heating phase (lasting approximately 2-3 min), which was followed by a thermal phase, when temperatures decreased from $45 \mathrm{MK}$ to $25 \mathrm{MK}$ over $10 \mathrm{~min}$.
\end{abstract}

Key words. Sun: chromosphere - Sun: corona - Sun: flares - Sun: magnetic fields - Sun: X-ray

\section{Introduction}

The interactions of magnetic structures and the conversion of magnetic free energy are commonly accepted as the main cause of solar flares. Despite many years of observational and theoretical investigations, several basic problems of solar flare physics are still not fully resolved. Even the apparently simple problem of evaluating the 3D structure of the interacting magnetic loops during solar flares can be understood only in a limited number of cases.

The 3D structure and evolution of the coronal magnetic field can be investigated indirectly by the analysis of the images of the various loops filled with emitting plasma or/and through a numerical model based on photospheric field measurements. The diverse topologies of

Send offprint requests to: P. Rudawy, e-mail: rudawy@astro.uni.wroc.pl the interacting magnetic loops have been described using three basic configurations, named $X, Y$ or $I$ by Sakai \& de Jager (1996). Analysing X-ray, ultraviolet and $\mathrm{H} \alpha$ observations of the C7.2 class solar flare in AR NOAA 8088 on 25 September 1997, we have previously built a comprehensive phenomenological 3D model (Paper I) of the magnetic configuration and its evolution. We have found that the flare was caused by two successive X-type interactions of magnetic loops. In the present paper, we compare this "observational" model with the results of the numerical modelling of the magnetic field. This comparison allows us to evaluate the level of agreement between these two methods of investigation of the coronal field of ARs and its evolution. We find that the correct interpretation and evaluation of the numerical results is difficult and can be considered as a typical problem. 


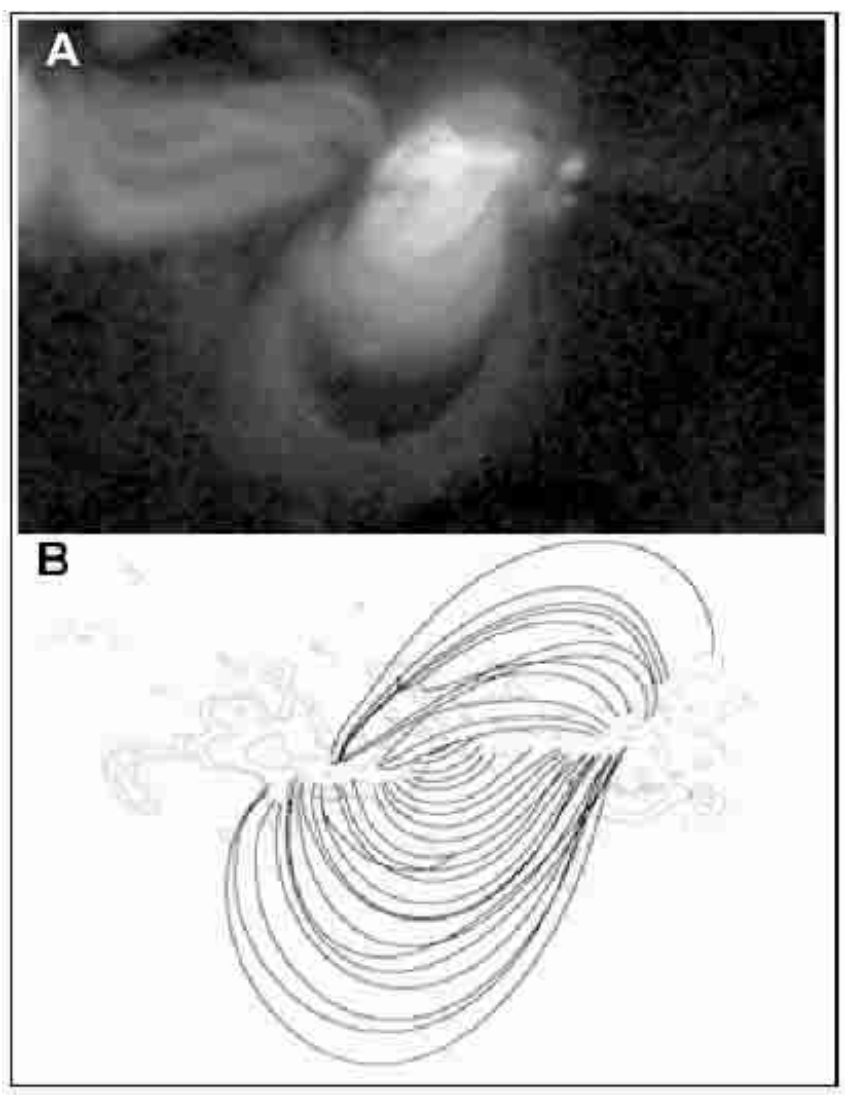

Fig. 1. Panel a) Yohkoh/SXT image of NOAA 8088 and NOAA 8087 active regions (central and left parts of the upper panel, respectively) taken after the flare on 26 September 1997. Panel b) Linear, force-free model of the magnetic field of the NOAA 8088 AR $\left(\alpha=0.01 \mathrm{Mm}^{-1}\right)$. The computed field lines show good global agreement with the bright structures presented in Panel A. The magnetic field isolines are: \pm 50 , $\pm 100, \pm 500, \pm 1000$ Gs (positive values shown with black lines, negative ones with grey lines). The size of the field of view is $250 \mathrm{Mm} \times 180 \mathrm{Mm}$ in the horizontal and vertical directions, respectively. North is up and West is to the right.

Using X-ray data taken with the RF15-I instrument on board the INTERBALL-TAIL satellite, we also extended our previous analysis of the relation between hard X-ray and microwave emission during the impulsive phase of the analysed flare, as well as analysis of temperature changes during the early phase of the flare. We also improve the previous estimation of the cooling time of the kernel observed in soft X-rays by the Yohkoh/SXT.

\section{Numerical model of the magnetic field of the active region NOAA 8088 on 25 September 1997}

The active region NOAA 8088 was observed on 25 September 1997 with many ground-based and satellite instruments, among others with the SOHO/MDI magnetograph. Using data obtained with this instrument, we computed the coronal magnetic field of this AR. The line of sight component of the photospheric magnetic field (observed one hour after the flare) was used as a boundary condition. The observed field was extrapolated to the corona using the discrete fast Fourier transform method under the linear force-free hypothesis $(\boldsymbol{\nabla} \times \boldsymbol{B}=\alpha \boldsymbol{B}, \alpha$ constant $)$, as proposed by Alissandrakis (1981). The studied AR was located at S28 E03, away from the solar disk centre, so we have used a transformation of coordinates from the observed frame to the local one, as discussed by Démoulin et al. (1997).

The results of the magnetic field modelling are affected by many factors: the limited spatial resolution of MDI data, changes in the photospheric magnetic field that may have occurred before the flare and the time at which the magnetogram was obtained, and mainly the limitations of the linear approach used. However, since transverse field measurements are not available, the approach used is the only suitable. The value of the free parameter $\alpha$, which measures the degree of the magnetic configuration shear, has been varied between 0 (potential field) and $0.03 \mathrm{Mm}^{-1}$ in order to achieve the best fit of the computed field lines to the loops observed by SOHO/EIT in $171 \AA$, $195 \AA$ and $284 \AA$ lines, as well as by Yohkoh/SXT.

As shown in Fig. 1, the global coronal field showed by the plasma of a few MK temperature can be considered as almost potential since it is best represented by field lines computed with $\alpha=0.01 \mathrm{Mm}^{-1}$. We have found that loops observed in different wavelengths (i.e. at different heights above the photosphere) could be fitted with different values of $\alpha$, in such a way that the magnetic shear seems to decrease with height (see Schmieder et al. 1996). Thus, we imply that the numerous low-lying, curved chromospheric threads, well observed in the high resolution $\mathrm{H} \alpha$ images of the AR, are not well represented by the low $\alpha$ value used for the model in Fig. 1.

We have also computed, following the method in Démoulin et al. (1996), the location of quasi-separatrix layers (QSLs). Titov et al. (1999) have introduced a new geometrical measure of QSLs giving a mathematically more grounded definition. The application of this new definition to observations has not yet been implemented. We apply here the earlier method which yields good accuracy in determining QSLs in observed magnetic configurations (see the review by Mandrini et al. 1997). It has been demonstrated both from a theoretical (Priest \& Démoulin 1995) and an observational (Mandrini et al. 1996; Démoulin et al. 1997; Gaizauskas et al. 1998; Bagalá et al. 2000) point of view that QSLs are likely places where magnetic reconnection can occur.

The lower panel in Fig. 2 shows the intersection of the computed QSLs with the photosphere. It can be seen that their locations and shapes agree in general with the locations of the plage brightenings observed in $\mathrm{H} \alpha$ before the flare and with the subsequent $\mathrm{H} \alpha$ flare kernels (see Paper I). The spatial agreement between QSLs and brightenings allows us to suppose that the later originated by magnetic reconnection occuring at QSLs before the flare. The released energy was then transported along the 
magnetic loops and deposited (at least partially) in the chromosphere at the location of the $\mathrm{H} \alpha$ brightenings.

After the careful study of our numerical results and the comparison with observations, we have found two possible scenarios to explain the evolution of the observed events.

\subsection{First scenario}

In the frame of the first scenario (which we will call "Scenario 1"), two sets of loops, existing before the flare, were involved in the energy release process before and during the flare. The continuous magnetic field lines in panel A of Fig. 3 would correspond to the field lines forming these loops. The first set of longer loops was rooted near brightenings B3 and B4. The second set of loops, shorter than the first one, had its footpoints close to brightenings B1 and B2. The photospheric magnetic field changed and was rebuilt during the $\mathrm{H} \alpha$ flare. This has been revealed by the eruption of a system of $\mathrm{H} \alpha$ threads described in Paper I. Therefore, the longer loops were forced to reconnect with the shorter ones. This was the origin of the preflare $\mathrm{H} \alpha$ plage brightenings and of the flare kernels, though the energy released should be smaller in the first case. After reconnection, two systems of reconnected loops appeared. The first system of very long, high loops connected brightenings $\mathrm{B} 1$ and $\mathrm{B} 4$ while the second system of much shorter loops connected B2 and B3 (see Fig. 3, panel B). The longer reconnected loops would correspond to the loops HL1 and HL2, which were observed in soft $\mathrm{X}$-rays (SXR) and were described in our phenomenological 3D model ("Model 0") in Paper I.

The SXR observations during the flare present two main peaks of the emission (see Paper I) originating in two independent sources. In this scenario we propose that there were two main energy release episodes, though energy was not only released during them. The energy release site was probably located at coronal heights above the longitudinal inversion line between the opposite polarities where B1 and B3 lay. The coronal configuration of the magnetic field suggests that the first energy release episode occurred in a X-type neutral ("quasi" neutral in our case) point (see Fig. 3, panels A and B, and the events described in Gaizauskas et al. 1998). This episode was the origin of the first intense SXR source, being the reconnection site above the very low and dense loops connecting B2 and B3. The location of that SXT source would coincide in projection with the location of the loops connecting B2 and B3. The energy released during the reconnection process flowed along the reconnected loops towards the chromosphere, induced the evaporation of chromospheric material which filled the loops with hot plasma and was the origin of the observed $\mathrm{H} \alpha$ flare kernels. The second episode of energy release occurred also at coronal heights in a similar way, but in that case the energy release site was located more to the south-west.

In the frame of this scenario, the very long loops (observed in SXR and called HL in Paper I) were the result of

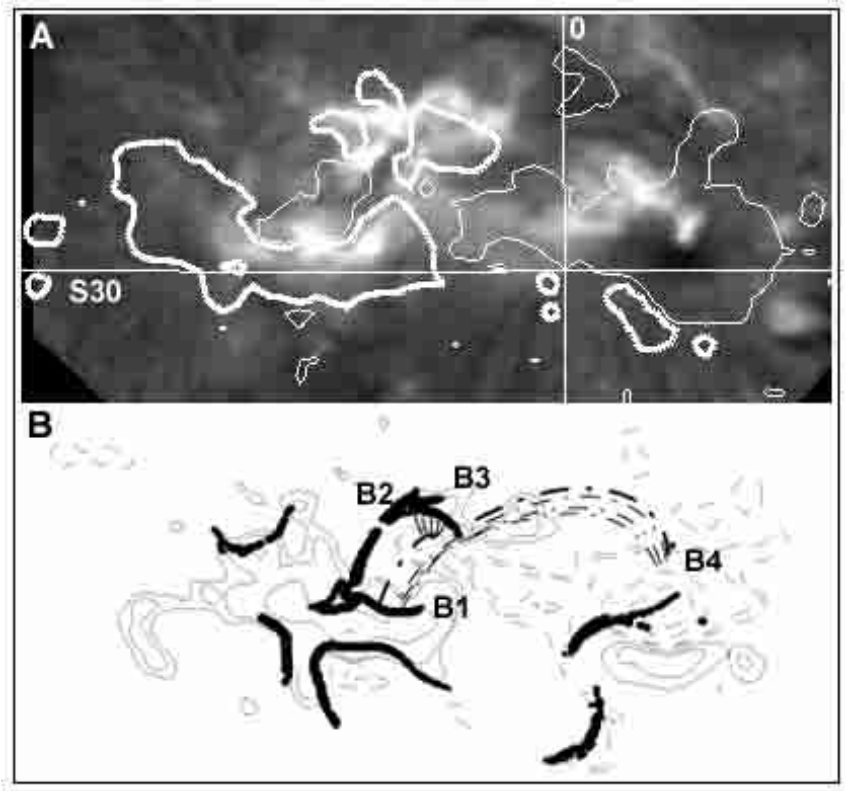

Fig. 2. Panel a) An image of the flare taken in the centre of the $\mathrm{H} \alpha$ line with the MSDP spectrograph at 12:09:25 UT with over-plotted SOHO/MDI magnetogram taken at 12:53:00 UT. The thick lines correspond to positive polarity magnetic fields while the thin lines to negative polarity ones. Panel b) Locations of the intersections of the QSLs (represented by bold, continuous lines) with the photosphere. The locations of these brightenings, where the flare kernels appeared, are approximately indicated by B1, B2, B3 and B4 labels. The shapes and locations of the QSLs agree well with the location of the $\mathrm{H} \alpha$ brightenings in the central and north-western parts of the active region. The positive polarity of the magnetic field is shown with continuous lines, negative one with dashed lines. The same sets of field lines as in panel b) of Fig. 3 are plotted from the observer's point of view.

magnetic reconnection. Unfortunately, in this scenario we cannot account for the role of another long and continuous loop observed with SXT, which we called LL in Paper I. According to our previous results, this loop played an important "active" role during the onset and evolution of the observed flare. In the scenario just discussed the role of this loop is completely "passive". LL, which was rooted to the east of B1 and B4 (see next section), could be the result of the reconnection process that went on during the preflare phase and that would not participate in the flare.

\subsection{Second scenario}

The first scenario does not fully account for the role of the structures observed in SXR (mainly the long loop LL). Therefore, we evaluated a second scenario for the flare, which we will call "Scenario 2". In this scenario we assume that a high and long system of stable loops (marked as HL in Fig. 4) stretched between the locations of brightenings B1 and B4 long before the onset of the flare (see Fig. 4).

These loops are well represented by our numerical model of the coronal field (see previous section) and can be 


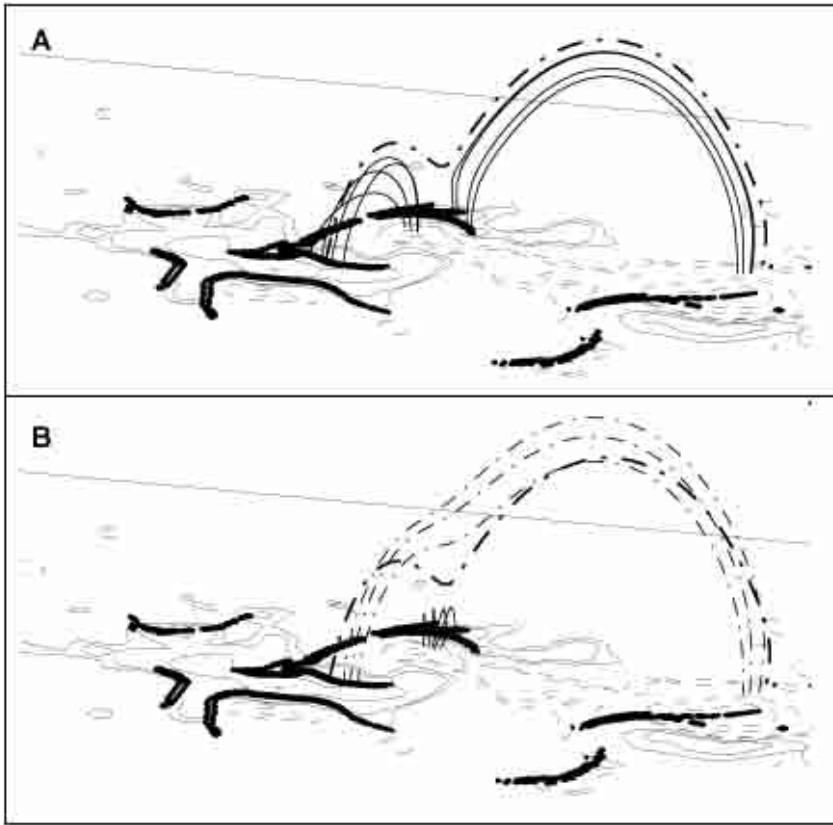

Fig. 3. Extrapolated coronal field lines related to the observed chromospheric and coronal features and the photospheric location of the QSLs over-plotted on the MDI magnetogram. The convention for the magnetic field is the same as in Fig. 1. We have chosen an arbitrary point of view so that the interacting field lines can be well visualised. Panel a) corresponds to field lines before reconnection. One magnetic field line (in thick dash-dotted style) belonging to the set of lines after reconnection has been added in order to show the topology around the probable reconnection region. Panel b) corresponds to field lines after reconnection.

recognised as the HL1 and HL2 loops described in our phenomenological "Model 0". As discussed above, these loops could be the result of a long and less energetic reconnection process occurring before the flare at QSLs, which was also the origin of the $\mathrm{H} \alpha$ plage brightenings. As was mentioned before, SXT recorded another long and continuous loop, which we called LL and described in Paper I. This loop was nearly perpendicular to the HL loops. In "Model 0 " we assumed that all these loops had been present before the flare in the AR. The LL loop could have been destabilised due to changes in the photospheric field and it could then have expanded. The X type interaction of LL with the higher lying HL loops was the origin of the first observed SXR source. Afterwards, the changes of the photospheric field, that were still going on, could cause the observed eruption of the $\mathrm{H} \alpha$ system of threads (see Paper I). This eruption could "push" the LL loop and force the second X type interaction (as discussed in Paper I). After a careful fitting of the computed field lines to the loops observed with SXT, we found in our numerical model a long, curved loop representing LL (see Fig. 4) stretched between the locations of brightenings B1 and B4 long befo) which was rooted close to brightenings B1 and B4. This loop stretched below the HL loops. Such a configuration of the computed field lines was very similar to the SXR loops (HL1, HL2 and LL) observed during the flare (see Fig. 4).
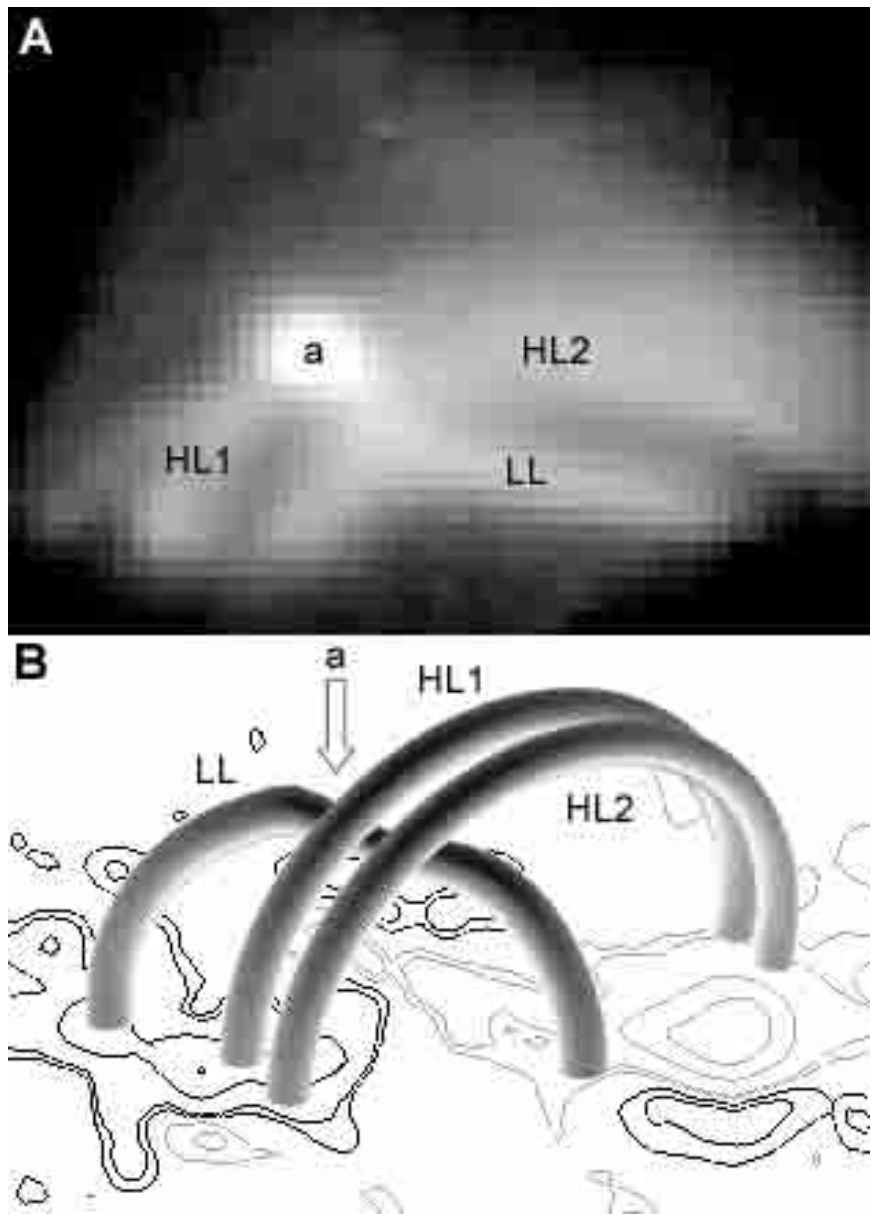

Fig. 4. Panel a) The SXT/Be119 image of the flare on 25 September 1997 averaged over time period between 12:06:38 UT and 12:13:46 UT. Logarithmic intensity scale is used. Panel b) Phenomenological 3D model of the flare ("Model 0") based on an analysis of the X-ray and $\mathrm{H} \alpha$ images and magnetograms. The interaction of the HL1 and HL2 loops with the raising LL loop triggered the flare at the point marked $a$.

According to Paper I, only a small fraction of the magnetic energy contained in the observed structures was released during the interactions that caused the flare. Thus, the loops survived the flare without any significant global changes. Similar to "Scenario 1", in this scenario the released energy flowed along the loops towards the chromosphere, induced chromospheric evaporation and was the origin mainly of the $\mathrm{H} \alpha$ flare kernels $\mathrm{B} 1$ and $\mathrm{B} 4$.

\subsection{Discussion of the scenarios}

After a comprehensive numerical modelling of the coronal magnetic field of the NOAA 8088 active region on 25 September 1997, we have found two possible scenarios to explain the observed flare and other associated phenomena. We stress that the computed magnetic model is the same for both scenarios.

"Scenario 1" could not account for the role of loop LL, observed as a unique structure in SXR. Thus, in the frame of this scenario we are forced to assume that LL was only 
a "passive" loop resulting from magnetic reconnection before the flare and not participating in it. In "Scenario 2" LL played an important role during the flare. It is modelled as a long curved loop rooted close to brightenings B1 and B4 and stretching below the HL loops. Such a configuration is in good agreement with the shape of the SXR observations described in Paper I (see Fig. 4).

In "Scenario 1" the energy was released by reconnection at a "quasi" neutral X-type point and the observed long SXR loops were the result of the reconnection process during the flare. In "Scenario 2" the energy was released due to an $\mathrm{X}$ type interaction between nearly perpendicular loops, which had existed before the flare and survived it without visible changes.

From our point of view, the first scenario corresponds to a solar flare occurring in a classical quadrupolar active region (see e.g. Gaizauskas et al. 1998). "Scenario 2" is a less "orthodox" scenario but agrees well with "Model 0", which is based only on observations (see Paper I). The second scenario, preferred by us, can explain well the observed SXR loops, the temporal sequence of the event, the connection of the flare with the eruption of the $\mathrm{H} \alpha$ system of threads and the presence of the second SXR source. The main problem of this scenario is that it cannot account for the presence of $\mathrm{H} \alpha$ flare kernels B2 and B3.

Taking into account our previous discussion, it is clear that both scenarios can explain the overall properties of the analysed flare. We think that a real scenario for the event can only be selected after comparing the numerical models with a broad set of multi-wavelengths observations. Even so, it may be difficult to evaluate the true scenario and that aspect seems to be a typical in the investigation of solar flares.

The solar flares can be observed in many spectral bands. X-ray, UV and magnetic observations are crucial to model the structure and evolution of the events. Since the magnetic field is not observed in the corona, photospheric magnetic data are the only available to infer through a numerical model the 3D configuration of the coronal field. $\mathrm{X}$-ray and UV images reveal projected configuration of the coronal magnetic loops filled with plasma and can be used to constrain numerical models. In order to obtain a better representation of the 3 -D coronal structure, vector magnetograms with higher spatial resolution and better temporal cadence would be needed. The same is valid in the case of X-ray and UV images since the spatial and temporal resolutions of the presently available observations are generally smaller than the processes going on during solar flares. Because the magnetic field in the region of the solar flare has a complex 3-D structure, the most useful $\mathrm{X}$-ray and UV data should be stereoscopic (collected by numerous (at least two) spacecrafts with preferably identical instruments on boards).

\section{Analysis of Hard X-ray and microwave observations of the flare}

In Paper I we briefly analysed the correlation between hard X-ray and $3 \mathrm{GHz}$ microwave fluxes, recorded during the impulsive phase of the analysed solar flare with the BATSE instrument on board of the GRO satellite and with the RT3 radiotelescope of the Ondřejov Astronomical Institute. The increase and oscillations of the $3 \mathrm{GHz}$ microwave flux were observed between 11:43 UT and 11:49 UT. The BATSE instrument recorded the hard Xray flux of the range of $25-50 \mathrm{keV}$ between 11:44:40 UT and 11:46:40 UT, when the detector was switched off (about two minutes before the drop of the radio flux to the level observed before the flare). The emission of the same flare was also recorded with the RF-15I instrument on board of the INTERBALL-TAIL satellite in many soft and hard X-ray channels. Using these data we analysed again the correlations between the hard X-ray and the $3 \mathrm{GHz}$ microwave fluxes during the whole period of the microwave emission. Exploring the possibilities offered by use of the RF-15I instrument, we evaluated also temperatures during the early phase of the flare.

\subsection{RF15-I instrument description}

The RF15-I Soft and Hard X-ray Photometer has been developed jointly by the Czech Astronomical Institute and the Space Research Centre of Polish Academy of Sciences (Sylwester et al. 2000). It was placed aboard the INTERBALL-TAIL spacecraft launched on 3 August 1995 and it operated continuously up to 16 October 2000. The photometer performed observations of the integrated X-ray flux in the energy range $2-240 \mathrm{keV}$.

The RF15-I X-ray photometer was equipped with two detector systems: the proportional argon gas-filled (350 Torr) detector of aperture of $4.5 \mathrm{~mm}^{2}$ equipped with a Be filter and the $\mathrm{Na}(\mathrm{I})$ scintillation detector of $15.2 \mathrm{~cm}^{2}$ aperture and of crystal thickness of $8 \mathrm{~mm}$.

The proportional detector nominally recorded the soft X-ray solar fluxes in three energy channels: 2-3, 3-5, 5-8 keV every $2 \mathrm{~s}$. From about mid-1997 the efficiency of the proportional detector started to decrease due to decrease of the gas pressure and ageing of the calibration source (for details see Sylwester et al. 2000).

The scintillation detector recorded the hard X-ray flux in five bands: $10-15,15-30,30-60,60-120,120-240 \mathrm{keV}$. In the lowest hard X-ray energy range (channel H1: 10-15 keV) the data were collected synchronously with the softer proportional detector channels every $2 \mathrm{~s}$. In the rest of the hard X-ray energy channels (H2 to H5 or $15-240 \mathrm{keV}$ ) the registration was performed every $0.125 \mathrm{~s}$.

\subsection{Observations}

Figure 5 presents the X-ray fluxes measured by the RF15-I photometer in five channels between 11:40 UT and 12:20 UT on 25 September 1997. The flare was recorded in 


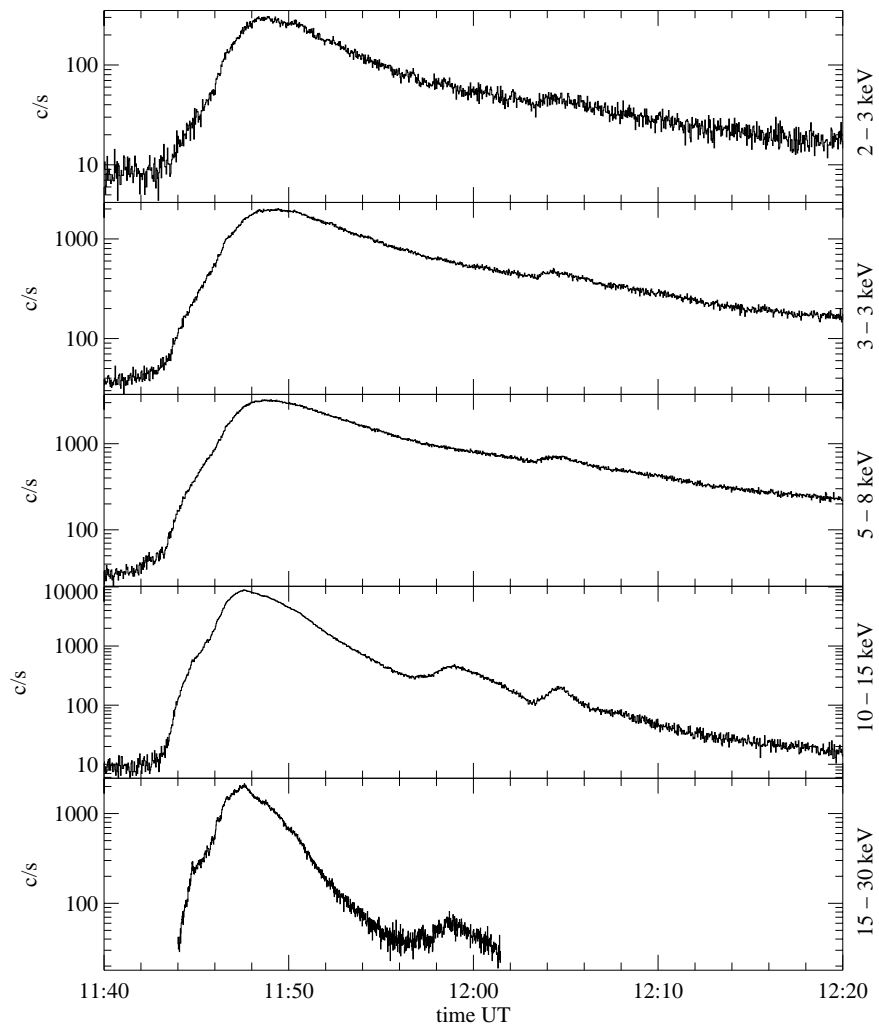

Fig. 5. Soft and hard X-ray fluxes recorded with the RF15-I instrument on board the INTERBALL-TAIL satellite on 25 September 1997 between 11:40 UT and 12:20 UT. The flux recorded with the $15-30 \mathrm{keV}$ channel is averaged over one second. The remaining four fluxes are averaged over two seconds.

all soft and two hard (10-15 and 15-30 keV) energy channels, but no emission was observed above $30 \mathrm{keV}$. In the soft energy channels, emission started at 11:43 UT, peaked at 11:49 UT and ended after 12:20 UT. In the first hard X-ray channel (H1), emission also started at 11:43 UT, but in the second hard channel (H2; 15-30 keV), emission was recorded only between 11:44 UT and 12:01 UT. In both hard channels the emission peaked approximately at 11:47 UT, two minutes before the peaks of the emission in the soft channels. In the H1 channel, two bumps emission were recorded at 11:59 UT and at 12:05 UT. The first bump was also recorded in the $\mathrm{H} 2$ channel while the second one was recorded in all soft channels. The instrument recorded also groups of peaks (spikes) before the maximum in both hard channels.

We rebinned the $\mathrm{H} 2$ and interpolated $\mathrm{H} 1$ data to a common time resolution of $1 \mathrm{~s}$. Using the transmission and response functions of the scintillation detector and assuming a thermal emission of the X-rays, we calculated the formal effective electron temperature and the emission measure from the ratio of the fluxes measured in two energy bands, even for the hard X-ray channels (see Fig. 6). The obtained values indicate that a group of pre-maximum temperature peaks could be emitted during the non-thermal heating phase (lasting approximately 2-3 min) followed by the thermal emission phase with

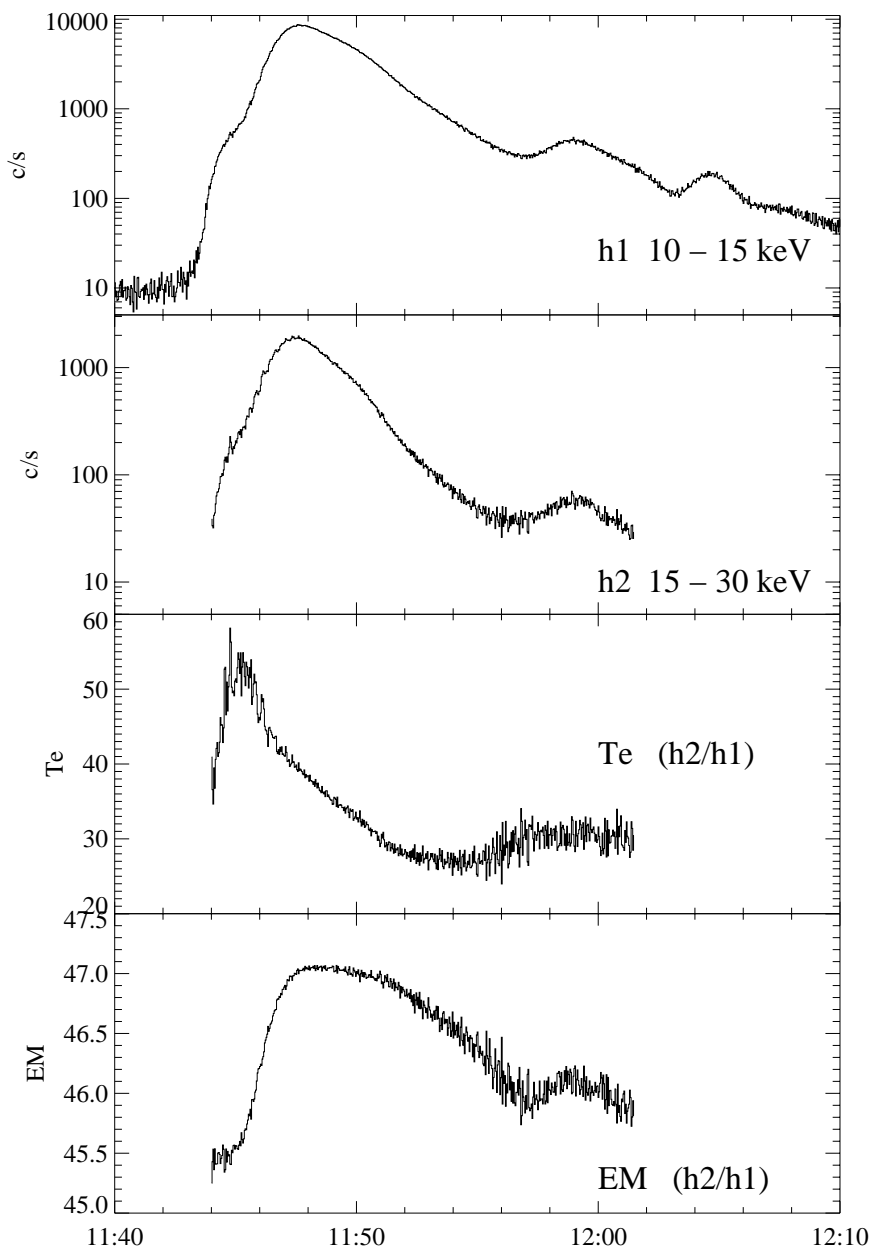

Fig. 6. Temporal changes of temperature $T_{e}$ and emission measure EM (two lower panels) calculated from the ratio of the fluxes taken with the H2 (15-30 keV) and H1 (10-15 keV) hard $\mathrm{X}$-ray channels of the RF15-I instrument (two upper panels).

temperatures decreasing from $45 \mathrm{MK}$ to $25 \mathrm{MK}$ during the next 8 to $10 \mathrm{~min}$.

Figure 7 shows the temporal changes of the ratios of the fluxes recorded in the $\mathrm{H} 2$ and $\mathrm{H} 1$ channels and in the S3 and S2 channels, respectively. The thermal heating phase of the flare started at about 11:50 UT, causing an increase in the $\mathrm{S} 3 / \mathrm{S} 2$ ratio, but at that time the non-thermal heating process had been still working. The thermal phase dominated after 11:54 UT. This means that the temperatures and emission measures of the emitting plasma were calculated for the period between 11:50 UT and 11:54 UT from mixed thermal and non-thermal radiation.

We also compared the data taken with the $\mathrm{H} 1$ and H2 channels of the RF15-I instrument and data taken with the RT3 radiotelescope. The raw observational data taken with all the instruments were smoothed using a $5 \mathrm{~s}$ wide boxcar-averaging filter (see Fig. 8). The smooth variations of the signals were removed to reveal the short period variations of the observed fluxes.

The eight main maxima of the X-ray fluxes, arbitrarily selected by us (marked A, B, D, E, H, K, L, O) are separated by approximately $44,36,33,59$ (27 plus 32 , taking 

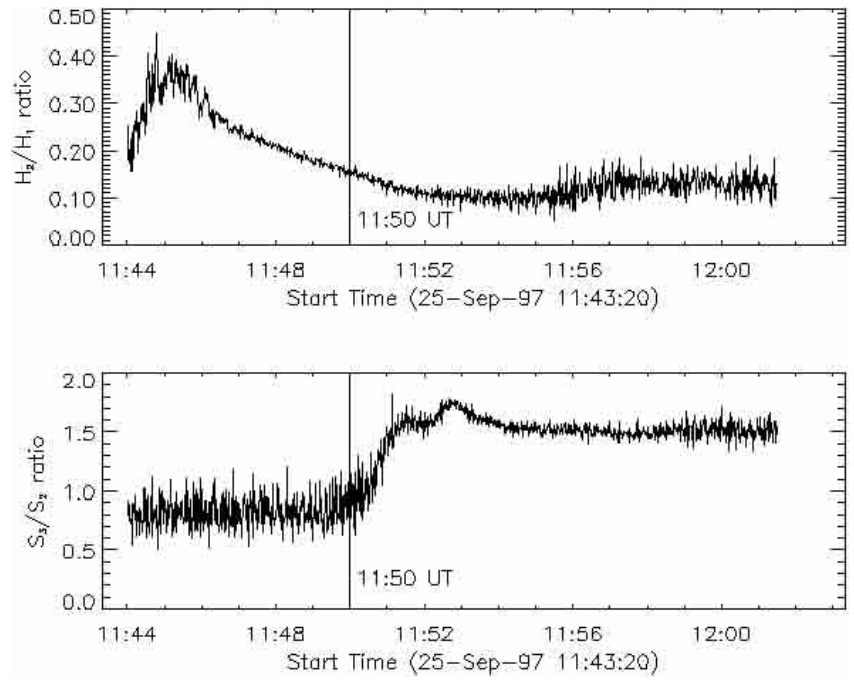

Fig. 7. Temporal changes of the ratios of the fluxes recorded in the $\mathrm{H} 2$ and $\mathrm{H} 1$ channels and in the $\mathrm{S} 3$ and $\mathrm{S} 2$ channels, respectively. The thermal heating phase of the flare started about 11:50 UT, causing an increase in the $\mathrm{S} 3 / \mathrm{S} 2$ ratio. At that time the non-thermal heating process had still been active. The thermal phase dominated after 11:54 UT.

into account the local maximum marked F), 44, 28 and 57 (31 plus 26, taking into account the local maximum $\mathrm{M})$ seconds. The microwave signal also reveals eight main maxima (marked A, B, D, E, G, K, L, N) separated by $44,36,33,46,57,35$ and 35 seconds. Periodograms of the three fluxes show that the changes are quasi-periodic. The statistically most significant period of X-ray oscillations is close to $30 \mathrm{~s}$. The overall changes of the microwave flux are very similar to the changes of the X-ray fluxes but the same maxima of the X-ray signal have no counterpart in the microwave signal (pulses marked $\mathrm{C}$ and $\mathrm{F}$ ). Interestingly, one strong local maximum $(G)$ of the microwaves appeared during a very small increase of the $\mathrm{X}$-ray flux.

The temporal variations of the fluxes recorded by the $\mathrm{H} 1$ and $\mathrm{H} 2$ detectors are very similar to each other but not identical. The correlation of the signals is $C_{12}=0.81$. It is very probable that both emissions were actuated by the same physical process but the localisation and temperatures of the both sources were different. The correlation of the signals recorded with the H1 detector and the RT3 radiotelescope is $C_{13}=0.38$ while the correlation between signals registered with the $\mathrm{H} 2$ and the RT3 is $C_{23}=0.43$.

The temporal variations of the analysed microwave and X-ray fluxes are consistent with the hypothesis that the energy of the analysed solar flare was produced in a turbulent kernel (Jakimiec \& Fludra 1991). The quasiperiodical changes of the signals could have been caused by series of small scale reconnections in many transient current sheets (Jakimiec et al. 1998). The observed time intervals between the local maxima were identical with relaxation times of the individual current sheets. The differences of the length of the intervals were caused by differences in local physical conditions. In addition, the

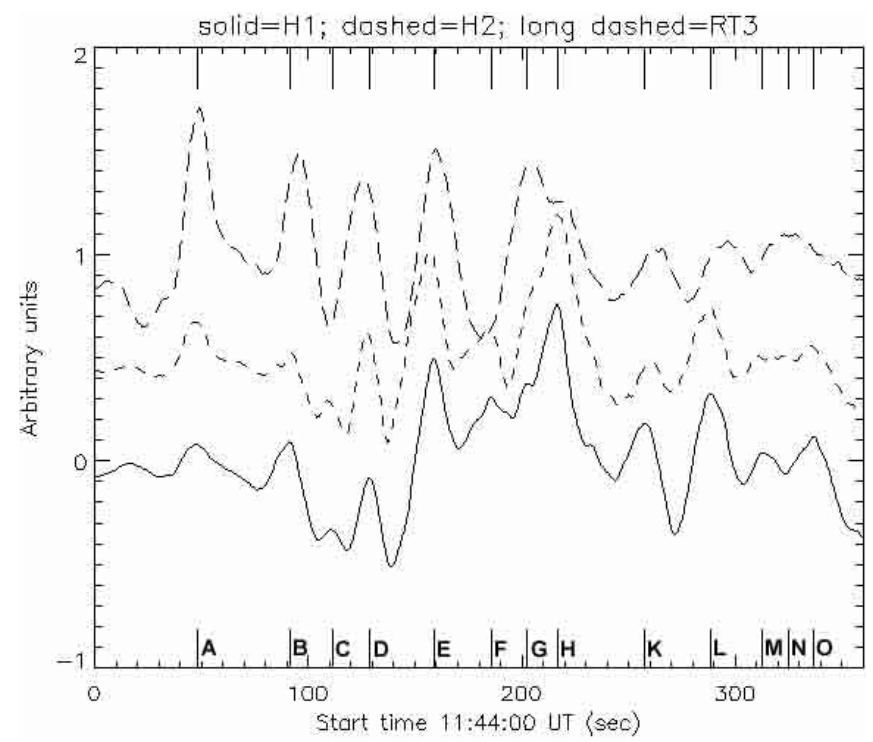

Fig. 8. Temporal variation of the hard X-ray fluxes taken with the $\mathrm{H} 1$ and the $\mathrm{H} 2$ channels of the RF15-I instrument and $3 \mathrm{GHz}$ microwave flux recorded with the $\mathrm{RT} 3$ radiotelescope of the Ondřejov Astronomical Institute.

slight differences of the temporal variations of the H1 and H2 HXR fluxes may be caused by the size and nonuniformity of the source.

\section{Conclusion}

The 3D structure and evolution of the magnetic field during solar flares can be investigated by an analysis of the 2D images of various magnetic structures filled with emitting plasma, and also through the numerical modelling of the coronal field of the active region based on the extrapolation of the measured photospheric field. While the numerical modelling of the magnetic field can give rise to more than one possible scenario for the observed event, a real scenario can only be selected after comparing the numerical model with various observational data.

Using hard X-ray and microwave data, we find that local pulses of these emissions, observed during the impulsive phase of the flare, show a tendency to a quasi-periodic repetition. Assuming thermal emission, we calculated the effective electron temperature with one second time resolution. The obtained values indicate that groups of premaximum peaks of the hard X-ray emission of the flare may be connected with the non-thermal heating phase (lasting approximately 2-3 min) followed by the thermal phase with temperatures decreasing from $45 \mathrm{MK}$ to $25 \mathrm{MK}$ during the next 8 to $10 \mathrm{~min}$.

Acknowledgements. We thank Prof. de Jager for many helpful comments during the redaction of the paper. PR has been supported by the grant No. 2 PO3D 00515 of the Polish Committee of Scientific Research. RF has been supported by the grant No. 2 PO3D 01614 of the Polish Committee of Scientific Research. C.H.M. is a member of the Carrera del Investigador Científico (CONICET). MS has been supported 


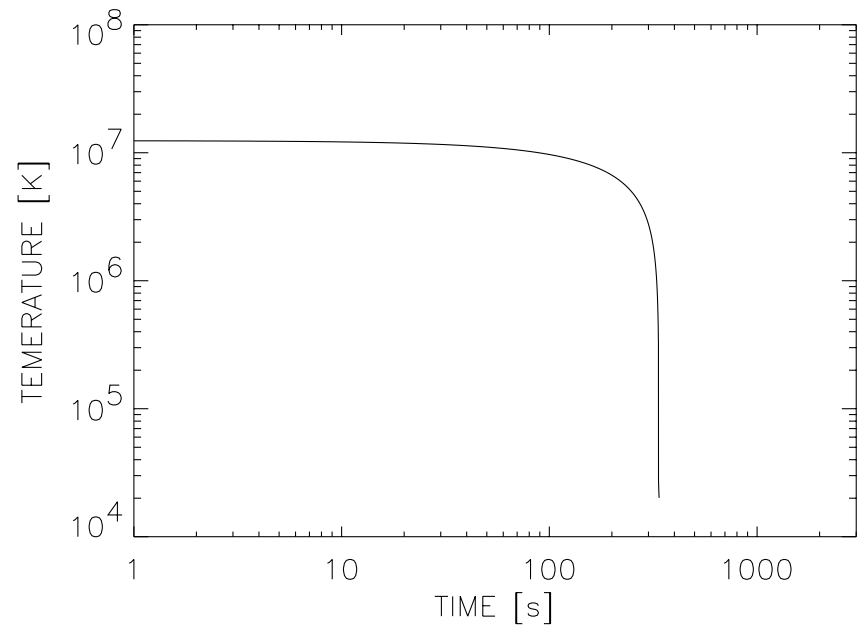

Fig. A.1. Cooling of the loop with semi-length $L=45500 \mathrm{~km}$, electron density $N_{\mathrm{e}}=2.9 \times 10^{11} \mathrm{~cm}^{-3}$ and initial temperature $T=12.4 \times 10^{6} \mathrm{~K}$.

by the grant No. 2.P03C.006.13 of the Polish Committee of Scientific Research.

\section{Appendix A:}

According to the X-ray GOES 9 data, the first interaction of the magnetic loops (source A) occurred at 11:40 UT and the first maximum of the thermal energy stored in the interacting loops occurred at 11:48 UT. We recorded the first $\mathrm{H} \alpha$ emission of the flare just before 11:50 UT. One can assume that the cooling time of the X-ray source is roughly equal to the time delay between the release of the energy and occurrence of the first $\mathrm{H} \alpha$ kernels of the flare.

In Paper I (Falewicz \& Rudawy 1999) we roughly estimated time necessary to cool down the hot X-ray loops to the temperature typical of $\mathrm{H} \alpha$ structures. The estimated cooling time of the A source by radiation was $T_{\mathrm{rad}} \approx 22 \mathrm{~s}$ while the cooling time by conduction was $T_{\text {cond }} \approx 4600 \mathrm{~s}$, i.e. two orders longer (van der Oort \& Mewe 1989). We concluded that the source was cooled mainly by radiation.

In estimating the loop cooling time by radiation anew we followed Švestka (1987). The temporal variation of kinetic temperature $T$ in such a loop is then schematically governed by the differential equation:

$\frac{\mathrm{d} T}{\mathrm{~d} t}=-\frac{\epsilon(T) n_{\mathrm{e}}^{2}+1.1 \times 10^{-6} T^{7 / 2} / L^{2}}{3 k n_{\mathrm{e}}}$,

where: $n_{\mathrm{e}}$ is the electron density, $L$ is the semi-length of the loop and $k$ the Boltzmann constant.

The radiative cooling function $\epsilon(T)$ depends only on temperature. This function can be approximated separately for different ranges of temperature. For $3 \times 10^{5}<T<3 \times 10^{7} \mathrm{~K}$ we use the same expression as Raymond et al. (1976):

$\epsilon(T) \simeq \frac{1.2 \times 10^{-19}}{T^{1 / 2}}$
For temperatures lower than $3 \times 10^{5} \mathrm{~K}$, down to $T \simeq 2 \times 10^{4} \mathrm{~K}$, we used the cooling function estimate as suggested by McClymont \& Canfield (1983):

$\epsilon(T) \simeq \frac{3.8 \times 10^{-37}}{T^{3}}$

The differential Eq. (A1) was solved numerically by the fourth-order Runge-Kutta method. Taking into account the evaluated physical parameters of the hot plasma: $T=12.4 \times 10^{6} \mathrm{~K}, E M=7.3 \times 10^{48} \mathrm{~cm}^{-3}$, $N_{\mathrm{e}}=2.9 \times 10^{11} \mathrm{~cm}^{-3}$ and a semi-length of the loop equal to $L=45500 \mathrm{~km}$ (Falewicz \& Rudawy 1999), we found that the cooling time was $T_{k}=340 \mathrm{~s}$ (see also in Fig. A.1), which is in good agreement with our observations.

We also estimated anew the time necessary to cool down the loop by conduction using a method proposed by de Jager et al. (1987). Taking into account the physical parameters of the hot plasma, calculated on the basis of the GOES 9 data: $T=12.4 \times 10^{6} \mathrm{~K}, N_{\mathrm{e}}=2.9 \times 10^{11} \mathrm{~cm}^{-3}$ and a semi-length of the hot X-ray source equal to $L=2800 \mathrm{~km}$ (Falewicz \& Rudawy 1999), we found that the conductive cooling time was $T_{\text {cond }} \approx 2500 \mathrm{~s}$. It is roughly similar to the previously estimated conductive cooling time and one order longer than the observed cooling time of the X-ray source.

\section{References}

Alissandrakis, C. E. 1981, A\&A, 100, 197

Bagalá, L. G., Mandrini, C. H., Rovira, M. G., \& Démoulin, P. 2000, A\&A, 363, 779

De Jager, C., Kuijpers, J., Correta, E., \& Kaufman, P. 1987, Sol. Phys., 110, 317

Démoulin, P., Bagalá, L. G., Mandrini, C. H., Hénoux, J. C., \& Rovira, M. G. 1996, A\&A, 325, 305

Démoulin, P., Hénoux, J. C., Priest, E. R., \& Mandrini, C. H. 1997, A\&A, 308, 643

Falewicz, R., \& Rudawy, P. 1999, A\&A, 344, 981

Gaizauskas, V., Mandrini, C. H., Démoulin, P., Luoni, M. L., \& Rovira, M. G. 1998, A\&A, 332, 353

Jakimiec, J., \& Fludra, A. 1991, Adv. Space Res., 11(5), 99

Jakimiec, J., Tomczak, M., Falewicz, R., Phillips, K. J. H., \& Fludra, A. 1998, A\&A, 334, 1112

Mandrini, C. H., Démoulin, P., van Driel-Gesztelyi, L., et al. 1996, Solar Phys., 174, 229

Mandrini, Démoulin, P., Bagalá, L. G., van Driel-Gesztelyi, L., et al. 1997, Sol. Phys., 174, 229

McClymont, A. N., \& Canfield, R. C. 1983, ApJ, 265, 483

Priest, E. R., \& Démoulin, P. 1995, J. Geophys. Res., 100, 2344

Raymond, J. C., Cox, D. P., \& Smith, B. W. 1976, ApJ, 204, 290

Sakai, J. I., \& de Jager, C. 1996, Space Sci. Rev., 77, 1-2

Schmieder, B., Démoulin, P., Aulanier, G., \& Golub, L. 1996, ApJ, 467, 881

Sylwester, J., Farnik, F., Likin, O., et al. 2000, Solar Phys. 197, 337

Švestka, Z. 1987, Solar Phys., 108, Letters

Titov, V. S., Démoulin, P., \& Hornig, G. 1999, Proc. of the Ninth European Meeting on Solar Phys., ed. A. Wilson, ESA-SP, 448, 715

van der Oort, G. H. J., \& Mewe, R. 1989, A\&A, 213, 245 